\section{MYC protein expression scoring and its impact on the prognosis of aggressive B-cell lymphoma patients}

This study examined the reproducibility of MYC and BCL-2 immunohistochemical scoring as well as the impact of higher expression of both proteins (double expressor status, DE) on survival and progression in a large retrospective cohort of aggressive B-cell lymphoma patients treated with rituximab plus cyclophosphamide, doxorubicin, vincristine and prednisone (R-CHOP) or RCHOP-like regimens with a median follow up of 67 months (range 0-138). We also investigated possible MYC protein expression cut offs with the highest reproducibility among pathologists and predictability of gene translocation. We showed that immunohistochemistry (IHC) for MYC and BCL-2 is highly reproducible when cut-off values of $>70 \%$ for MYC and $>50 \%$ for BCL- 2 are used. This threshold not only predicts the presence of rearrangements (with respect to $M Y C$ ), but is also clinically valuable. In fact, it identifies a subset of patients who are poor responders and who may benefit from alternate therapeutic strategies.

Several investigators have demonstrated that diffuse large B-cell lymphomas (DLBCL) with MYC and BCL-2 double expression (DE-LBCL) have adverse prognosis when treated by conventional immunochemotherapies. ${ }^{1}$ Therefore, evaluation of MYC and BCL-2 protein expression by IHC is an important tool in the prognostic stratification of patients. ${ }^{2}$ Although expression of MYC-IHC in $\geq 40 \%$ neoplastic cells and the BCL- 2 in $\geq 50 \%$ have been indicated as prognostically significant cut offs in many reports, among hematopathologists, some disagreement about these thresholds remains. ${ }^{3,4}$ Standardizing cut offs to define positivity for MYC-IHC and BCL-2-IHC with higher reproducibility and prognostic impact is thus highly desirable in order to optimize patient management. ${ }^{5,6}$
We analyzed 753 aggressive B-cell lymphoma patients. These included: Burkitt lymphomas (BL, $n=223)$; DLBCL not otherwise specified (DLBCL-NOS, $n=456$ ); high grade B-cell lymphomas (HGBCL) with $M Y C$ and $B C L-2$ and/or BCL-6 rearrangement [HG double hit (HGDH), $\mathrm{n}=51$, including 8 blastoid, 16 diffuse monomorphic and 27 intermediate morphology]; and HGBCL, NOS $(n=23) .^{7-9}$ All cases belonging to these categories, except for BL, will be referred to hereon as non-BL. The following clinical data were collected for non-BL: sex, age at diagnosis, ECOG performance status, stage (Ann Arbor), lactate dehydrogenase (LDH) level, International Prognostic Index (IPI), bulky disease, extranodal versus nodal, bone marrow involvement. Original MYC-IHC and BCL-2-IHC slides were re-evaluated by 3 trained pathologists blinded as to the original scores. Although aware that immunohistochemical interpretation may be affected by technical issues, we intentionally decided not to re-stain all slides in only one laboratory, but instead to confront original slides from different laboratories which apply different unmasking procedures and use different clones on a "real-life" basis (Table 1). Scores were reported in $5 \%$ intervals rounded up to the nearest $10 \%$. Any discrepancies in MYC-IHC and BCL-2-IHC scoring was resolved by consensus on a multi-head microscope until an agreement of $>95 \%$ concordance was reached. Discrepancy was defined as $10 \%$ deviation. Our findings confirm that BCL-2-IHC scoring is highly reproducible across the different institutions and that a $\geq 50 \%$ cut off is reliably assessable [agreement $>90 \%$; $=0.97$; standard error (SE): $=0.018$; 95\% confidence interval (CI): 0.765 0.901]. On the contrary, scoring MYC-IHC staining was confirmed to be critical, mainly because of the variability of staining intensity, percentage of positivity, presence of necrosis and crush artifacts. Concordance was high only for MYC-IHC positivity $>70 \%$, while a larger discrepancy was observed in the range $40-69 \%{ }^{10}$ (Figure 1 ). The latter can have a crucial impact on clinical decision making

Table 1. Immunohistochemical and FISH analysis of MYC* and BCL-2* proteins and genes in the different institutions.

\begin{tabular}{|c|c|c|c|}
\hline & MYC clone/source & MYC antigen retrieval/dilution BCL-2 clone/source & BCL-2 antigen retrieval/dilution \\
\hline Berlin & $\begin{array}{c}\text { EP121, } \\
\text { Epitomics, Germany }\end{array}$ & $\begin{array}{c}\text { Pressure cooker, } \\
\text { citrate buffer } \mathrm{pH} 6.0\end{array}$ & $\begin{array}{l}\text { Pressure cooker, } \\
\text { citrate buffer pH } 6.0\end{array}$ \\
\hline Bologna & EP121, Epitomics, Milan & PT link $92^{\circ} \mathrm{C} / 1: 100$ & PT link $90^{\circ} \mathrm{C} / 1: 100$ \\
\hline Firenze & Y69, Roche, Milan & SP124, Roche, Milan & Ready to use \\
\hline London & Y69, Abcam, UK & 124, Dako, UK & PT link $90^{\circ} \mathrm{C} / 1: 100$ \\
\hline Siena \& Nairobi & Y69, Roche, Milan & Ready to use $\quad$ SP124, Roche, Milan & Ready to use \\
\hline & MYC BAP probe/source & MYC FUSION probe/source & BCL-2 BAP probe/source \\
\hline Berlin & $\begin{array}{c}\text { Split Signal MYC, Agilent, } \\
\text { Germany }\end{array}$ & $\begin{array}{l}\text { Dual fusion IGH/MYC, Zytomed, } \\
\text { Germany }\end{array}$ & $\begin{array}{c}\text { Splits Signal BCL-2, Agilent, } \\
\text { Germany }\end{array}$ \\
\hline Bologna & $\begin{array}{l}\text { ZytoLight SPEC MYC Dual Color } \\
\text { Break Apart Probe, Bio-Optica, } \\
\text { ZitoVision GmbH, Germany }\end{array}$ & $\begin{array}{c}\text { ZytoLight SPEC MYC/IgH } \\
\text { Dual Color Dual Fusion Probe, Bio-Optica, } \\
\text { ZitoVision GmbH, Germany }\end{array}$ & $\begin{array}{l}\text { ZytoLight SPEC BCL-2 Dual Color } \\
\text { Break Apart Probe, Bioptica, } \\
\text { ZitoVision GmbH, Germany }\end{array}$ \\
\hline Firenze & $\begin{array}{l}\text { ZytoLight SPEC MYC Dual Color } \\
\text { Break Apart Probe, Bio-Optica, } \\
\text { ZitoVision GmbH, Germany }\end{array}$ & $\begin{array}{c}\text { ZytoLight SPEC MYC/IgH Dual Color } \\
\text { Dual Fusion Probe, Bio-Optica, } \\
\text { ZitoVision GmbH, Germany }\end{array}$ & $\begin{array}{l}\text { ZytoLight SPEC BCL-2 Dual Color } \\
\text { Break Apart Probe, Bioptica, } \\
\text { ZitoVision GmbH, Germany }\end{array}$ \\
\hline London & Kreatech, Leica, UK & Kreatech, Leica, UK & Kreatech, Leica, UK \\
\hline
\end{tabular}




\section{Concordance of MYC IHC scoring: original versus revised}

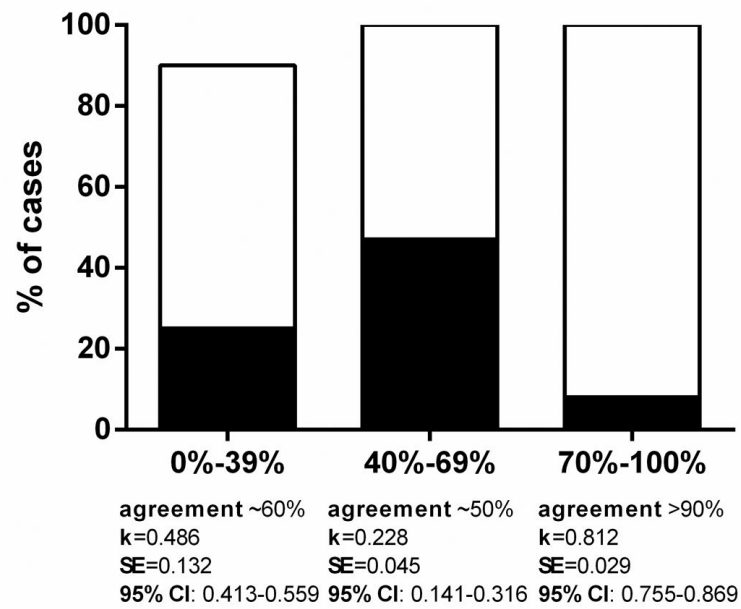

$\square$ Concordance
Non Concordance

Figure 1. Graph of original and revised MYC immunohistochemistry (IHC) scores. The percentage of cases with concordance (white bars) or discordance (black bars) after MYC IHC revision for the three ranges is shown. The concordance analysis results are listed. SE: standard error; $\mathrm{Cl}$ : confidence interval.

and patient care, as well as on the comparison between different studies. Therefore, we propose the threshold of MYC-IHC $70-100 \%$ to define non-BL cases with high expression of MYC and $0-39 \%$ to classify samples with low expression of MYC protein. Samples with MYC-IHC scores between $40 \%$ and $69 \%$ could not be categorized as MYC protein negative or positive with acceptable accuracy in routine practice and should be termed as cases with intermediate MYC expression. Moreover, we suggest scoring should preferably be defined on moderately to strongly stained nuclei and in hot spot areas, if present. After revision, in the non-BL series, prevalence of DE-LBCL as defined by the cut offs proposed by the literature (MYC-IHC $\geq 40 \%$ and BCL-2-IHC $\geq 50 \%$ ) was $32 \% \quad(n=176 / 530) ;^{7}$ of these 100 showed MYC-IHC $\geq 70 \%$ (DE $70 \%$ in the following).

A second end point of this study was to investigate the relation between MYC-IHC and MYC gene alterations, trying to identify parameters potentially applicable in a value-for-money approach. When using the threshold of MYC-IHC $\geq 80 \%$ in BL and $\geq 70 \%$ in non-BL, around $99 \%$ $\mathrm{BL}$ and $88 \%$ non-BL cases with $M Y C$ rearrangements can be predicted. Therefore, considering the high correlation between MYC gene rearrangement and MYC protein expression in BL, FISH analysis could theoretically be limited to cases with atypical morphology and/or immunophenotype and MYC-IHC $<80 \%$. In non-BL, a cut off of MYC-IHC $\geq 70 \%$ would identify cases at higher probability of MYC rearrangement. However, it should be noted that roughly $10 \%$ of $\mathrm{HGDH}$ have a lower expression of MYC; in these cases, FISH should be recommended. ${ }^{11}$

The third end point was to perform a survival analysis in cases of $\mathrm{HGDH}, \mathrm{DE}$, nonDH/nonDE patients for whom overall survival (OS) and progression-free survival (PFS) data were available. HGDH lymphomas are known to be very aggressive neoplasms that do not respond to standard therapy and they have a dismal prognosis. ${ }^{12}$ However, it has recently been reported that the adverse prognosis may be mitigated by other factors such as lack of BCL-2 protein, and absence/low MYC protein expression. ${ }^{5}$ The clinical significance of MYC-IHC and BCL-2IHC expression has been extensively studied but the results have not been consistent; hence, there is still no clear use for protein expression evaluation in the clinic. $^{13,14}$

Univariate Cox analysis showed that the factors significantly associated with OS and PFS were: age [Odds Ratio (OR): $4.8 \%$ each year; confidence interval (CI): 1.03-1.066; $P<0.001]$, LDH $>150$ UI/L (OR: $187 \%$, CI: 1.756-4.715; $P<0.001$ ), IPI (OR: $71 \%$, CI: 1.422-2.056; $P<0.001$ ), bone marrow involvement (OR: $86.2 \%, \mathrm{CI}$ : 1.102-3.145; $P=0.02$ ), MYC translocation (OR: 71\%, CI: 1.281-2.297; $P<0.001$ ), $B C L-2$ translocation (OR: 97\%, CI: $1.389-2.795 ; P=0.002$ ), MYC-IHC $\geq 70 \%$ (OR: $65 \%$, CI: $0.22-0.539 ; P<0.001$ ), DH status (OR: $310 \%$, CI: 2.529-6.662; $P<0.001$ ), DE $70 \%$ status (OR: $61.5 \%$, CI: 1.976-2.67; $P=0.04)$. Stepwise multivariate analysis demonstrated that IPI (OR: 62.2\%, CI: 1.225-2.146; $P=0.001$ ), MYC-IHC $\geq 70 \%$ (OR: $224.8 \%$, CI: $1.714-$ 6.155 ; $P<0.001$ ), DH status (OR: $131.7 \%$, CI: $1.003-$ 5.350; $P=0.049$ ), DE $70 \%$ status (OR: $177.5 \%$, CI: $1.020-$ 1.080; $P=0.006)$ were independent prognostic factors that influence patients' outcome.

A comparison of HGDH, DE-LBCL [according to World Health Organization (WHO) criteria, i.e. MYC-IHC $\geq 40 \%$ and BCL-2-IHC $\geq 50 \%$ ] and nonDH/nonDE subsets of non-BL in terms of OS and PFS confirmed that $\mathrm{HGDH}$ patients showed significantly worse survival than the other two groups $\left(P<0.001 ; \chi^{2} 39.24\right.$ for OS and 13.13 for PFS) (Figure 2A and Online Supplementary Figure S1A).

By stratifying DE-LBCL according to MYC protein expression $(70-100 \%, 40-69 \%$, ) the prognosis turned out to be poorer in the former $\left(P<0.001, \chi^{2} 13.152\right.$ for OS and 10.723 for PFS) (Figure 2B and Online Supplementary Figure $S 1 B)$.

Dividing HGDH cases according to MYC protein positivity revealed that those with MYC-IHC $\geq 70 \%$ had a worse prognosis than cases with MYC-IHC $\leq 69 \%$ $\left(P<0.001, \chi^{2} 48.215\right.$ for OS and 35.549 for PFS) (Figure 2C and Online Supplementary Figure S1C). This supports prior observations that patients with $M Y C$ rearrangements or DH lymphomas devoid of MYC-IHC expression might exhibit less aggressive clinical behaviors. ${ }^{13}$

Given that the highest concordance and reproducibility of MYC-IHC was $>70 \%$, we compared OS and PFS of DE-LBCL showing MYC-IHC $>70 \%$ and BCL-2-IHC $>50 \%$ with HGDH cases; we found no significant differ- 
A

OVERALL SURVIVAL IN DOUBLE HIT versus DOUBLE EXPRESSOR versUS NON DOUBLE HIT NON DOUBLE EXPRESSOR

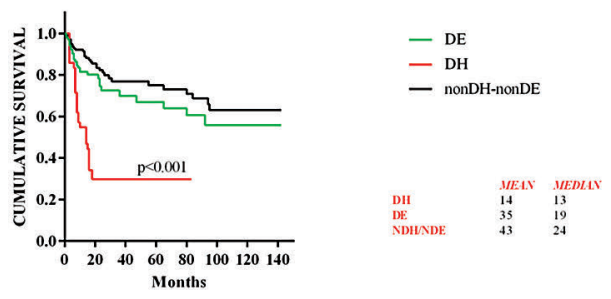

C

OVERALL SURVIVAL IN DOUBLE HIT DEPENDING ON MYC PROTEIN EXPRESSION

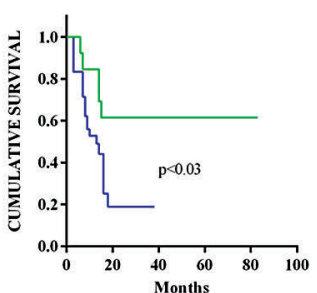

- DHMYC 0.69\%

— DHMYC $70-100 \%$

D

OVERALL SURVIVAL IN DOUBLE HIT versus DOUBLE EXPRESSOR with MYC 70-100\% versus NON DOUBLE HIT NON DOUBLE EXPRESSOR

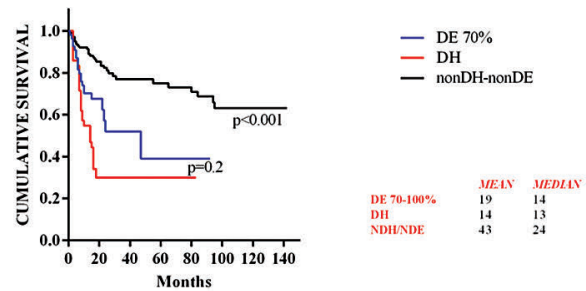

Figure 2. Kaplan-Meier curves of overall survival (OS) in the different patient groups. (A) Kaplan-Meier curve of OS in double hit (DH) versus double expressor (DE) versus non-double hit/non-double expressor (nonDH/nonDE) lymphomas. There was a significant difference in OS between the DH and the other two groups (log rank test, $P<0.001$ ). DH lymphomas were defined by having both MYC and BCL-2 rearrangements; DE were characterized by showing MYC-immunohistochemistry $(\mathrm{IHC}) \geq 40 \%$ and $\mathrm{BCL}-2-\mathrm{IHC} \geq 50 \%$; nonDH/nonDE included cases without double hits or with one only single hit, and with none or only one protein expression between MYC and BCL-2. (B) Kaplan-Meier curve of OS in DE depending on MYC protein expression. OS was significantly lower in the group showing MYC-IHC $\geq 70 \%$ than in the group with MYC-IHC 40-69\% (log rank test, $P<0.001$ ) indicating that a MYC-IHC $\geq 70 \%$ cut off defines a clinically relevant subgroup of DE cases. (C) Kaplan-Meier curve of OS in DH depending on MYC protein expression. There was a significant difference in OS between the two groups (MYCIHC $0-69 \%$ and $\mathrm{BCL}-2-\mathrm{IHC} \geq 50 \%$, and MYC-IHC $\geq 70 \%$ and $\mathrm{BCL}-2-\mathrm{IHC} \geq 50 \%$ ) with $\mathrm{MYC} \geq 70 \%$ cases having the lower survival rate (log rank test, $P<0.001$ ). Noteworthy, among HGDH with MYC-IHC 0-69\%, the 3 cases showing MYC-IHC $<40 \%$ had a mean OS of 44 months (data not shown). These findings indicate that adverse survival in HGDH is highly impacted by high MYC-IHC expression ( $\geq 70 \%$ ). (D) Kaplan-Meier curve of OS in DH versus DE as defined by MYC-IHC $\geq 70 \%$ and $\mathrm{BCL}-2-\mathrm{IHC} \geq 50 \%$ versus nonDH/nonDE. OS overlaps in $\mathrm{DH}$ and DE with MYC-IHC $\geq 70 \%$ and $\mathrm{BCL}-2-\mathrm{IHC} \geq 50 \%$ (Iog rank test, $P=0.2$ for $\mathrm{DH}$ versus $\mathrm{DE}$ with $\mathrm{MYC} \geq 70 \%$ and $\mathrm{BCL}-2-\mathrm{HHC} \geq 50 \%$;), this is worse than the OS of the nonDH/nonDE (log rank test, $P<0.001$ for either DH or DE with MYC $\geq 70 \%$ and $\mathrm{BCL}-2$ $\mathrm{IHC} \geq 50 \%$ versus nonDH/nonDE).

ences in prognosis $\left(P=0.2, \chi^{2} 0.777\right.$ for OS and 3.441 for PFS) (Figure 2D and Online Supplementary Figure S1D).

Interestingly, the OS and the PFS of HGBCL, NOS strictly depended on MYC expression being 30 and 28 months in HGBCL, NOS with MYC 40-69\%, 10 and 14 months in those with MYC 70-100\%.

These findings again highlight the need to establish more widely reproducible cut-off values for IHC assays with higher predictive value, to safely stratify high-risk patients. Moreover, they may also, at least in part, explain the above mentioned inconsistencies in published data.

Finally, since the cell-of-origin (COO) classification of LBCL exerts a prognostic impact, with non-GCB lymphomas showing a poorer prognosis than GCB ones, we stratified HGDH and DE-LBCL according to COO defined by Hans' algorithm. ${ }^{15} \mathrm{~A}$ poorer prognosis was demonstrated only in patients with MYC-IHC $\geq 70 \%$, with no differences according to the COO. However, no conclusions can be drawn due to both the limited number of cases examined per group and to the IHC assessment of $\mathrm{COO}$ which is known to misclassify a subset of samples.

Different molecular mechanisms may be responsible for the same disease entity and neoplastic phenotype. FISH currently represents the gold standard for identifying rearrangements, but it is not capable of detecting genetic deregulation affecting gene expression at tran- scriptional and post-transcriptional levels that might result in protein overexpression and neoplastic transformation. ${ }^{4}$ Our results support the role of MYC protein as the active trigger of the MYC-mediated oncogenic effects since protein expression levels likely represent a more direct measure of the activity of a particular gene $e^{16}$ and MYC-IHC should be undertaken in all cases. However, the last WHO criteria assessed that MYC staining is not reliable enough to select cases for FISH analysis. ${ }^{7}$ According to our results, the cut off of MYC-IHC $\geq 70 \%$, based both on reproducibility among pathologists and clinical impact, is a good indicator of underlying $M Y C$ gene rearrangement. Nonetheless, our promising data need further validation in an independent cohort of patients. In addition, given the highest scoring efficiency, the cut offs of MYC-IHC $\geq 70 \%$ and BCL-2-IHC $\geq 50 \%$ could be applied when FISH is not available (due to issues of practicability, cost, short turn-around-time, quality control) to select a subset of patients who are poor responders and who may benefit from alternate therapeutic strategies.

Maria R. Ambrosio, ${ }^{1}$ Stefano Lazzi, ${ }^{1}$ Giuseppe Lo Bello, Raffaella Santi, Leonardo Del Porro, ${ }^{2}$ Maria M. de Santi, Raffaella Guazzo, ${ }^{1}$ Lucia Mundo, ${ }^{1}$ Luigi Rigacci, ${ }^{4}$

Sofia Kovalchuck, ${ }^{4}$ Noel Onyango, ${ }^{5}$ Alberto Fabbri, ${ }^{6}$

Emanuele Cencini, ${ }^{6}$ Pier Luigi Zinzani, ${ }^{7}$ Francesco Zaja,

Francesco Angrilli, ${ }^{9}$ Caterina Stelitano, ${ }^{10}$ Maria G. Cabras, ${ }^{11}$

Giuseppe Spataro, ${ }^{12}$ Roshanak Bob,,$^{13}$ Thomas Menter, ${ }^{14,15}$ 


\section{Massimo Granai, ${ }^{1}$ Gabriele Cevenini, ${ }^{1}$ Kikkeri N. Naresh, ${ }^{15}$ Harald Stein, ${ }^{13}$ Elena Sabattini ${ }^{16}$ and Lorenzo Leoncini ${ }^{1}$}

${ }^{1}$ Department of Medical Biotechnologies, University of Siena, Italy; ${ }^{2}$ Division of Pathological Anatomy, University of Florence, Italy; ${ }^{3}$ Unit of Pathological Anatomy, AOU Siena, Italy; ${ }^{4}$ Hematology Division, AOU Careggi, University of Firenze, Italy; ${ }^{5}$ Department of Clinical Medicine and Therapeutics, Unit of Medical Oncology, University of Nairobi, Kenya; ${ }^{6}$ Haematology Unit, Azienda Ospedaliera Universitaria Senese, Siena, Italy; 'Institute of Hematology "L. e A. Seràgnoli", University of Bologna, Italy; ${ }^{8}$ Clinica Ematologica ed Unità di Terapie Cellulari "Carlo Melzi", DAME, University of Udine, Italy; "Ematologia Ospedale Civile dello Spirito Santo, Pescara, Italy; ${ }^{10}$ Ematologia, Ospedale Bianchi Melacrino Morelli, Reggio Calabria; ${ }^{11}$ Hematology, Cagliari Hospital, Italy; ${ }^{12}$ Post Graduate School of Public Health, University of Siena, Italy; ${ }^{13}$ Pathodiagnostik Lab. Berlin, Germany; ${ }^{14}$ Institute of Pathology and Medical Genetics, University Hospital of Basel, Switzerland; "15epartment of Cellular and Molecular Pathology, Hammersmith Hospital Campus, Imperial College Healthcare NHS Trust, London, UK and ${ }^{16}$ Unit of Haemolymphopathology, Department of Hematology and Oncology, University Hospital of Bologna, Italy

Acknowledgments: the Authors would like to thank additional hematological centers that contributed with clinical data: Di Raimondo F. - Hematology, Catania University, Italy, Cimino G. and Centra N. Hematology, Latina Hospital, Galieni P. - Hematology, Ascoli Piceno Hospital, Martelli M. and Di Rocco A. - Hematology, Roma University "La Sapienza", Italy, Vigna E. - Hematology, Cosenza Hospital, Italy, Taratini G. - Hematology, Barletta Hospital, Italy, Scalone R. - Hematology, casa di Cura La Maddalena, Palermo, Italy, Mannina D. - Hematology, Papardo Hospital, Messina, Italy

Correspondence:lorenzo.leoncini@dbm.unisi.it doi:10.3324/haematol.2018.195958

Information on authorship, contributions, and financial \& other disclosures was provided by the authors and is available with the online version of this article at www. haematologica.org.

\section{References}

1. Sarkozy C, Traverse-Glehen A, Coiffier B. Double-hit and doubleprotein-expression lymphomas: aggressive and refractory lymphomas. Lancet Oncol. 2015;16(15):e555-567.

2. Miura K, Takahashi H, Nakagawa M, et al. Clinical significance of co-expression of MYC and BCL-2 protein in aggressive B-cell lymphomas treated with a second line immunochemotherapy. Leuk.
Lymphoma. 2016;57(6):1335-1341.

3. Kluk MJ, Ho C, Yu H, et al. MYC Immunohistochemistry to Identify MYC-Driven B-Cell Lymphomas in Clinical Practice. Am. J. Clin. Pathol. 2016;145(2):166-179.

4. Mahmoud AZ, George TI, Czuchlewski DR, et al. Scoring of MYC protein expression in diffuse large B-cell lymphomas: concordance rate among hematopathologists. Mod. Pathol. 2015;28(4):545-551.

5. Kawashima I, Inamoto Y, Maeshima AM, et al. Double-Expressor Lymphoma Is Associated with Poor Outcomes after Allogeneic Hematopoietic Cell Transplantation. Biol Blood Marrow Transplant. 2018;24(2):294-300.

6. Rosenthal A, Younes A. High grade B-cell lymphoma with rearrangements of MYC and BCL-2 and/or BCL6: Double hit and triple hit lymphomas and double expressing lymphoma. Blood Rev. 2017; 31(2):37-42

7. Swerdlow S.H, Campo E, Harris NL, et al. WHO classification of Tumours of Haematopoietic and Lymphoid, $4^{\text {th }}$ ed. Lyon, France, IARC Press, 2017.

8. Naresh KN, Hazem AHI, Lazzi S, et al. Diagnosis of Burkitt Lymphoma using an algorithmic approach applicable in both resource-poor and resource-rich countries. Br J Haematol. 2011, 154(6):770-776

9. Menter T, Medani H, Ahmad R, Flora R, Trivedi P, Reid A, Naresh $\mathrm{KN}$. MYC and BCL2 evaluation in routine diagnostics of aggressive B-cell lymphomas - presentation of a work-flow and the experience with 248 cases. Br J Haematol. 2017;179(4):667-688.

10. Landis JR, Koch GG. The measurement of observer agreement for categorical data. Biometrics. 1977;33(1):159-174.

11. Scott, DW, King RL, Staiger AM, et al. High-grade B-cell lymphoma with MYC and BCL2 and/or BCL6 rearrangements with diffuse large B-cell lymphoma morphology. Blood. 2018;131(18):2060-2064.

12. Friedberg JW. How I treat "Double Hit" lymphoma. Blood. 2017; 130(5):590-596.

13. Herrera AF, Mei M, Low L, et al. Relapsed or Refractory DoubleExpressor and Double-Hit Lymphomas Have Inferior ProgressionFree Survival After Autologous Stem-Cell Transplantation. J Clin Oncol. 2017;35(1):24-31.

14. Green TM, Young KH, Visco C, et al. Immunohistochemical doublehit score is a strong predictor of outcome in patients with diffuse large B-cell lymphoma treated with rituximab plus cyclophosphamide, doxorubicin, vincristine, and prednisone. J Clin Oncol. 2012;30(28):3460-3467.

15. Staiger AM, Ziepert M, Horn H, et al. Clinical Impact of the Cell-ofOrigin Classification and the MYC/ BCL-2 Dual Expresser Status in Diffuse Large B-Cell Lymphoma Treated Within Prospective Clinical Trials of the German High-Grade Non-Hodgkin's Lymphoma Study Group. J Clin Oncol. 2017;35(22):2515-2526.

16. Ott G, Rosenwald A, aCampo E. Understanding MYC-driven aggressive B-cell lymphomas: pathogenesis and classification. Blood. 2013; 122(24):3884-3891. 